\title{
MAPPING THE TOTAL GRAVITATING MASS IN THREE GROUPS OF GALAXIES
}

\author{
THIERING I. ${ }^{1}$, DAHLEM M. ${ }^{2}$ \\ 1 Landessternwarte, Heidelberg, Germany \\ ${ }^{2}$ STSCI, Baltimore, U.S.A.
}

We report on the detection and analysis of hot ionized gas in three groups of galaxies (MKW 4s, AWM 5, NGC 6329 group) at distances above $100 \mathrm{Mpc}$ (recession velocities above $8000 \mathrm{~km} \mathrm{~s}^{-1}$ ). In all three cases their emission is centered on the dominant member of the group (NGC 4104, NGC 6269, NGC 6329, respectively). The central electron densities of the gas $n_{\mathrm{e}, 0}$ are about $0.02 \mathrm{~cm}^{-3}$, decreasing to the outside as: $n_{\mathrm{e}}=n_{\mathrm{e}, 0}\left[1+\left(r / r_{\mathrm{c}}\right)^{2}\right]^{-3 \beta / 2}$, with an exponent $\beta=0.40$.. 0.77 , (see Tab. 1). In each case, the spectra are well-represented by thermal Raymond-Smith spectra with $\mathrm{k} T \simeq 1.0$ to $1.3 \mathrm{keV}$ in the center and a weak increase by ca. $0.5 \mathrm{keV}$ to the outside, indicating a slight cooling flow. The fits suggest that none of the central galaxies hosts an AGN that contributes significantly to the emission in the ROSAT band ( 0.1 to $2.4 \mathrm{keV})$. Using the radial temperature and density distributions, the total gravitating mass within a radius $r$ can be derived. This value is $1.7 \pm 0.410^{13} \mathrm{M}_{\odot}$ within $300 \mathrm{kpc}$ for all three cases, as opposed to to $10^{14 . .15} \mathrm{M}_{\odot}$ for rich clusters.

TABLE 1. Results of the imaging and the spectral fits

\begin{tabular}{|c|c|c|c|}
\hline group: & MKW4s & AWM5 & NGC 6329 gr. \\
\hline $\begin{array}{l}\text { cent.surf.br. } \Sigma_{0}\left[\frac{\mathrm{cts}}{\sigma^{\prime \prime}}\right] \\
\text { core radius } r_{\mathrm{c}}[\mathrm{kpc}] \\
\text { exponent } \beta \\
L_{\mathrm{x}, \text { tot }}\left[10^{42} \mathrm{erg} / \mathrm{s}\right]\end{array}$ & $\begin{array}{l}0.072 \pm 0.005 \\
11.0 \pm 1.1 \\
0.47 \pm 0.002 \\
4.72\end{array}$ & $\begin{array}{l}0.047 \pm 0.007 \\
9.4 \pm 1.4 \\
0.40 \pm 0.002 \\
7.85\end{array}$ & $\begin{array}{l}0.068 \pm 0.005 \\
18.7 \pm 2.2 \\
0.77 \pm 0.09 \\
1.90\end{array}$ \\
\hline $\begin{array}{l}\text { spectral fit: } \chi^{2} / \nu \\
N_{\mathrm{Hgal}}^{\#}\left[10^{20} \mathrm{~cm}^{-2}\right] \\
z_{\text {inner }} / z_{\odot} \\
z_{\text {outer }} / z_{\odot} \\
\mathrm{k} T_{\text {center }}[\mathrm{keV}] \\
\mathrm{k} T_{\text {outer }}[\mathrm{keV}]\end{array}$ & $\begin{array}{l}0.85 \\
1.81 \\
0.78 \pm 0.34 \\
0.05 \pm 0.06 \\
1.28 \pm 0.12 \\
1.80 \pm 0.48\end{array}$ & $\begin{array}{l}1.00 \\
5.32 \\
0.58 \pm 0.43 \\
(0.26 \pm 0.31) \\
1.19 \pm 0.14 \\
1.6 \pm 0.3\end{array}$ & $\begin{array}{l}1.16 \\
1.24 \\
1.49 \pm 0.55 \\
0.06 \pm 0.02 \\
1.02 \pm 0.07 \\
1.25 \pm 0.20\end{array}$ \\
\hline $\begin{array}{l}n_{\mathrm{e}, 0}\left[\mathrm{~cm}^{-3}\right] \\
\text { cooling time } t_{\mathrm{br}}\left[10^{9} \mathrm{yr}\right] \\
\text { cooling radius } r_{\mathrm{cool}}[\mathrm{kpc}] \\
\text { accreting mass } \dot{M}\left[\mathrm{M}_{\odot} / \mathrm{yr}\right] \\
M_{\text {grav }}(<\mathrm{r}=300 \mathrm{kpc})\left[\mathrm{M}_{\odot}\right]\end{array}$ & $\begin{array}{l}0.020 \\
1.4 \\
51 \\
6.1 \\
210^{13}\end{array}$ & $\begin{array}{l}0.013 \\
2.1 \\
42 \\
6.5 \\
1.510^{13}\end{array}$ & $\begin{array}{l}0.022 \\
1.1 \\
59 \\
5.4 \\
210^{13}\end{array}$ \\
\hline
\end{tabular}

